\title{
IMAGE ANALYSIS APPROACHES TO IMPROVE THE THIN LAYER CHROMATOGRAPHY - CHEMOMETRIC-BASED INVESTIGATIONS OF NATURAL EXTRACTS
}

\author{
MARIA SIMIONa , SIMONA CODRUTA AURORA COBZAC ${ }^{a *}$, \\ DORINA CASONI ${ }^{\mathrm{a}}$
}

\begin{abstract}
The combination of high-performance thin-layer chromatography (HPTLC) with image analysis (IA) and chemometrics becomes an attractive tool for natural extracts investigations. The large variability of these samples requires powerful image acquisition devices, multivariate image processing techniques and advanced chemometric methods to facilitate the interpretation of the chromatographic data. In the current study, two image acquisition devices and different image processing procedures were investigated using the HPTLC chromatograms of hydroalcoholic extracts of Gallium verum. Different sets of chromatographic data were generated for both UV chromatograms (obtained at $254 \mathrm{~nm}$ and at $366 \mathrm{~nm}$ ) using images acquired with a digital camera and an UV-Vis TLC scanner. In all cases the Principal Component Analysis (PCA) technique was used in order to extract the information from chromatographic profiles. Variables of gray and pure colour red, green and blue intensities of pixels from start to front were used as input data in all cases. The results obtained by PCA investigations of HPTLC data from UV chromatograms at $254 \mathrm{~nm}$ and $366 \mathrm{~nm}$ respectively, provided complementary information related to the characteristics of the investigated extracts. Moreover, important steps as appropriate color scale selection and image processing/analysis procedures were pointed out based on the obtained results.
\end{abstract}

Keywords: Thin-Layer Chromatography, Image Analysis, Principal Component Analysis, Gallium verum extracts

a Babeş-Bolyai University, Faculty of Chemistry and Chemical Engineering, 11 Arany Janos, RO-400028, Cluj-Napoca, Romania.

*Corresponding author: csimona@chem.ubbcluj.ro 


\section{INTRODUCTION}

Thin layer chromatography (TLC) is a very popular analytical technique currently used to investigate the number of components in a mixture, verify the identity of substances, quantify numerous compounds in various foods, pharmaceuticals and biological samples and monitor the progress of reactions. Nowadays, due to the advantages offered by the improvements regarding sample application technique, image acquisition systems, computer controlled scanning instrument and image analysis software, high performance TLC combined with image analysis (HPTLC-IA) is feasible to analyze the compounds with overlapping chromatograms [1], quantify the fluorescent compounds [2], investigate the antiradical activity of plant materials [3], screening the compounds from natural extracts $[4,5]$ and fingerprinting analysis of various plants material $[6,7]$. Among the numerous application of HPTLC, the fingerprint analysis is of special interest. In this area, chemometric techniques for HPTLC data evaluation were recently employed [8, 9]. Chromatographic data can be obtained from the chromatograms by the means of classical densitometry, or by processing the image obtained with flatbed scanner or different digital cameras [10-12]. Several software packages such as ImageJ, Just TLC, Sorbfil TLC Videodensitometer and others can be used for image processing [13-17]. Once the chromatographic data are properly recorded, extracted, and pre-treated, multivariate data analysis methods such as principal component analysis (PCA), hierarchical cluster analysis (HCA) or linear discriminant analysis (LDA) can be applied to extract the most important chemical information which lead to samples classification or to detect the classification patterns [18-21]. The increasing scientific interest to combine HPTLC with multivariate data analysis is a promising research field in herbal analysis. In this area, more investigations related to the image acquisition and image processing techniques are necessary in order to extract the meaningful information from obtained chromatograms.

In this context, the present paper focused on transformation of HPTLC chromatogram of Galium verum extracts into numerical data appropriate to chemometric analysis using various image analysis procedures of images acquired with two different systems. In our investigation, extracts of Gallium verum (Lady's Bedstraw) were used as test model. The plant, currently involved in traditional folk medicine, can be easily recognized by its distinct sunny yellow color and tiny little flowers [22-24]. As a remedy, Lady's Bedstraw is used for skin problems (burns, psoriasis), kidney and bladder stones and epilepsy (due to its sedative effect). Also it is used to curd milk and as a yellow food dye. The beneficial effects of Gallium verum extracts are generally attributed to the biological active compounds as iridoides, flavonoids, antrakinnone, alkane and 
essential oils which are present in the plant $[25,26]$. Due to this large variety of compounds, the hydroalcoholic extracts of Gallium verum were considered as a good input data for image processing and multivariate analysis investigations.

The aim of the study is to highlight the most appropriate steps in image processing prior to application of multivariate data analysis in order to differentiate various Gallium verum hydroalcoholic extracts based on their HPTLC fingerprints. For a better understanding of extracts classification, the available red, blue, green and gray channels will be used for chromatograms digitization. Also the study aims to identify the factors that lead to extracts classification according to their provenience and extraction system.

\section{RESULTS AND DISCUSSION}

Extracts from natural products are complex mixtures that contain a large number of compounds. Usually thin-layer chromatographic profiles are taken into consideration for quality evaluation or authenticity assays. In most of the cases different chemometric approaches are used in order to extract the valuable information from chromatographic profile. In the current study, the Principal Component Analysis (PCA) technique was used as an alternative way to differentiate various Gallium verum hydroalcoholic extracts based on image analysis (IA) of their HPTLC fingerprints. Different aspects related to the image acquisition and processing techniques of chromatograms obtained with two different detection approaches (fluorescence quenching under $254 \mathrm{~nm}$ and fluorescence at $366 \mathrm{~nm}$ respectively) were employed in order to provide complementary information on hydroalcoholic extracts characteristics.

\section{HPTLC fingerprint of Gallium Verum extracts}

The HPTLC fingerprint analysis based on images obtained under UV light investigation at $254 \mathrm{~nm}$ and $366 \mathrm{~nm}$ after spraying with NTS was used to seek for characteristic patterns of Gallium verum extracts of different origin using the advantages of multivariate image processing and principal component analysis (PCA) tool.

Hydroalcoholic extracts of Gallium verum vegetal material commercialized by Plafar (P) and Dacia (D) manufacturers (Romania), obtained with different composition of extracting system $(60 \%-100 \%$ ethanol) were investigated. A normal-phase chromatographic system, using a HPTLC Silica gel $60 F_{254}$ plates and the developing solvent mixture composed of ethyl acetate: toluene: formic acid: water (30:1.5:4:3 v/v), was employed to separate highly and medium polar phenolics and obtain chromatographic bands with improved shapes. 
The HPTLC chromatograms (Fig.1) obtained under $254 \mathrm{~nm}$ and respectively $366 \mathrm{~nm}$ (images acquired with digital camera) revealed differences between $\mathrm{D}$ and $\mathrm{P}$ samples especially in upper area. Moreover, the investigation under $366 \mathrm{~nm}$ revealed that the extracts are rich in some phenolic compounds with a pattern dominated by blue and red color bands.

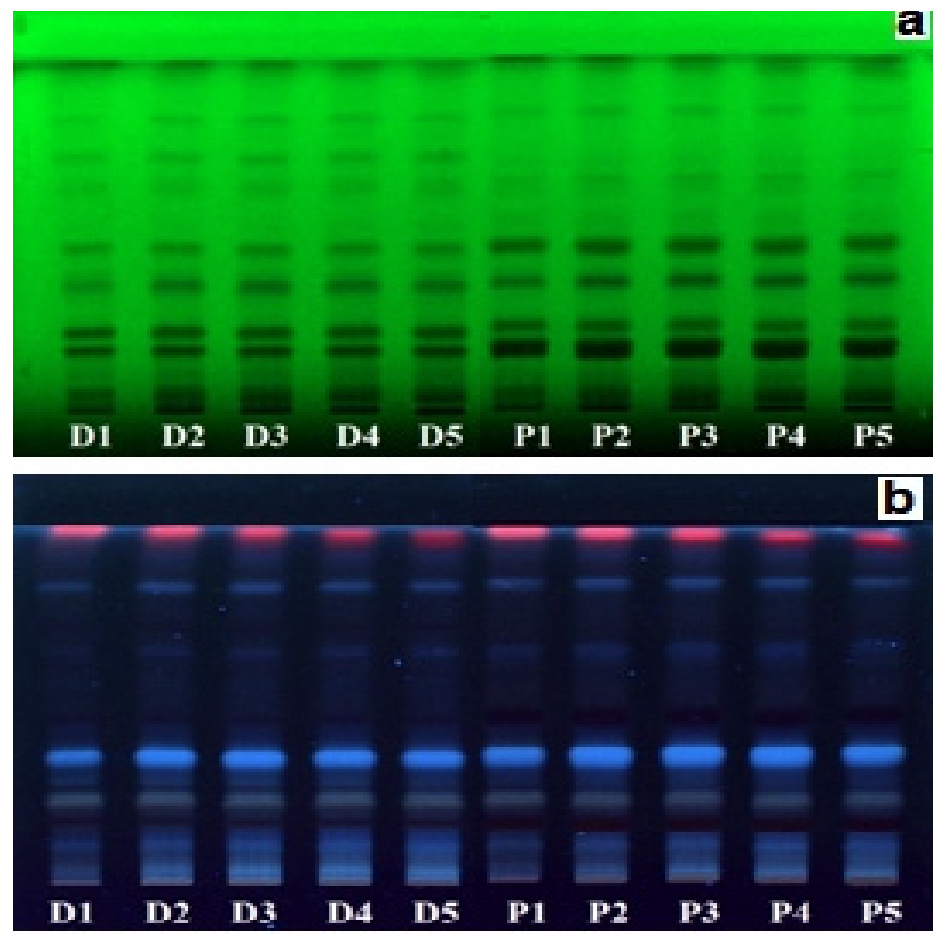

Figure 1. HPTLC chromatograms of Gallium verum extracts obtained using HPTLC Silica gel $60 \mathrm{~F}_{254}$ chromatographic plates and mixture of ethyl acetate - toluene formic acid - wather (30:1.5:4:3, v/v) as mobile phase: (a) detection at $\lambda=254 \mathrm{~nm}$; (b) detection at $\lambda=366$ after spraying with NTS (D1-D5 extracts of Gallium verum vegetal powder commercialized by Dacia manufacturer using 100\%, $90 \%, 80 \%$ $70 \%$ and $60 \%$ ethanol solutions; P1-P5 extracts from Gallium verum vegetal powder commercialized by Plafar manufacturer using 100\%, 90\%, $80 \% 70 \%$ and $60 \%$ ethanol solutions)

Based on these observations, different image processing approaches have been applied for a complete evaluation of the chromatographic profile of analyzed samples. Image processing through the gray (Gy), red $(R)$, green $(\mathrm{Gn})$ and blue $(\mathrm{B})$ scales were used in order to increase the detection selectivity and differentiate between compounds according to their fluorescent colors and quantity found in the investigated extracts. 


\section{Data acquisition and chemometric analysis}

Different sets of chromatographic data were generated for both UV chromatograms (obtained at $254 \mathrm{~nm}$ and at $366 \mathrm{~nm}$ ) using images acquired by a digital camera and an UV-Vis TLC scanner device. In all cases, the HPTLC chromatograms were digitized using the TLC Analyser software and selection of the gray, red, green and blue scales respectively. According to the different colour channels used for digitizing the HPTLC chromatograms, the obtained profiles show different maximum and minimum values at the same $R_{f}$ values (Fig. 2). This finding revealed that all of the scales contribute with important complementary information related to characteristic aspects of investigated extracts. Moreover, it could be observed that in case of fluorescence quenching, green and gray scales seems to provide the most significant quantity of information related to the investigated extracts.
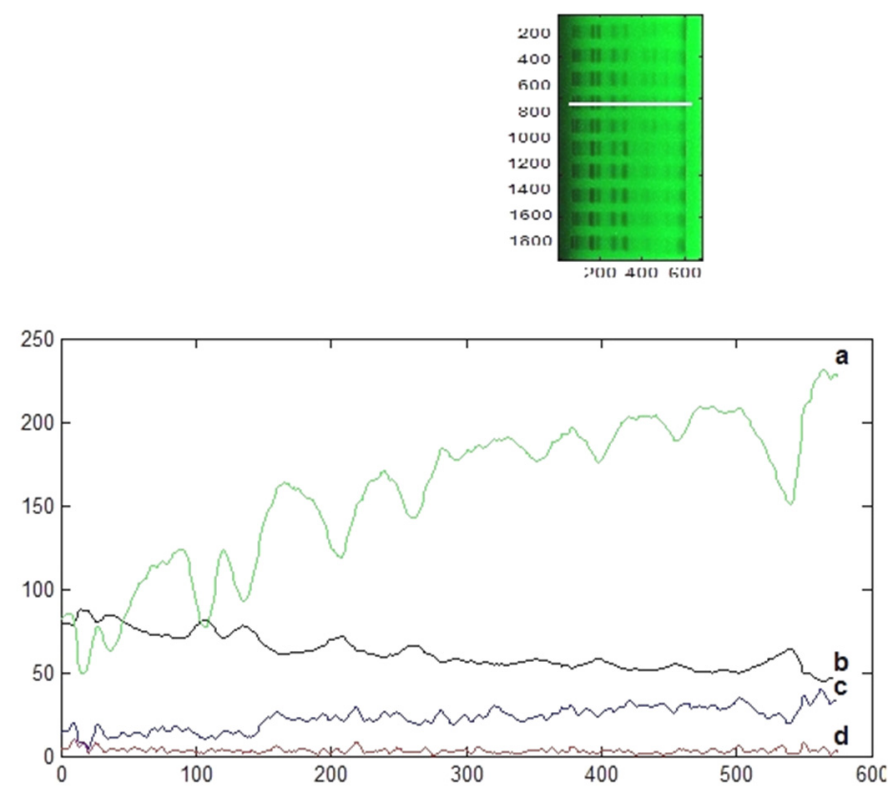

Figure 2. Digitized HPTLC chromatograms obtained by processing the UV image (fluorescence quenching) of chromatographic plate using TLC Analyser software on different colour scale: (a) green (Gn); (b) gray (Gy); (c) blue (B); (d) red (R) scale

For more insights, the PCA technique was applied on data matrices represented by numerical values of gray and pure RBG color intensity (as independent variables) corresponding to the associated $R_{F}$ values. Based on the PCA investigations, the most significant results are discussed as follows. 
Using the image acquired under UV light at $\lambda=254 \mathrm{~nm}$ by digital camera, the first principal component (PC1, representing $93.89 \%$ of the total variance in the data set) was associated with the upper edge of the plate $\left(R_{f}=0.975\right)$ while the second PC (PC2, accounting $5.96 \%$ from the data variability) was associated with the bottom area of the plate $\left(R_{f}=0.02\right)$. Considering also the third PC (PC3, accounting only $0.100 \%$ of data variability) the area associated to the middle of the plate $\left(R_{f}=0.44\right)$ could be investigated. By a graphical representation of the first two PCs (Fig. 3) no significant differences were revealed between information provided by red and blue scale selection while each of the gray and green scales account for supplementary information on the investigated extracts. Moreover, by using the green scale for investigation, a good separation of the extracts according to the provenience (commercial manufacturers) could be observed.

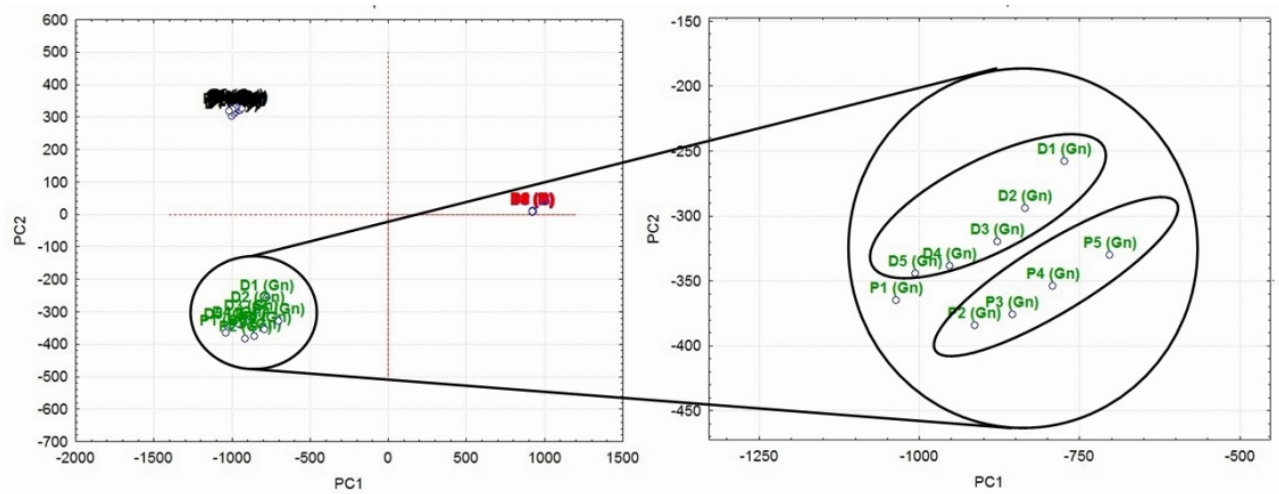

Figure 3. PCA classification of the investigated extracts (according to PC1 vs. PC2 representation) based on chromatographic fingerprints provided by UV254 image (acquired with digital camera) digitized through the gray (Gy) and red (R), blue (B), green $(G n)$ color scale selection

Considering the contribution of the PC3, the representation PC1 vs. PC3 (Fig. 4) shows, on green and gray scale, a good separation of the investigated samples according to their provenience.

The PCA investigations of the data matrices, provided by TLC image (fluorescence quenching) acquired with the scanner device revealed that the first three PCs explain $99.82 \%$ of the total variability. The PC1 variable (accounting for $96.22 \%$ of data variability) was associated with the upper area of the plate $\left(R_{f}=0.97\right.$ ) while the PC2 (accounting for $3.43 \%$ of data variability) was associated with the bottom area of the plate $\left(R_{f}=0.03\right)$. 


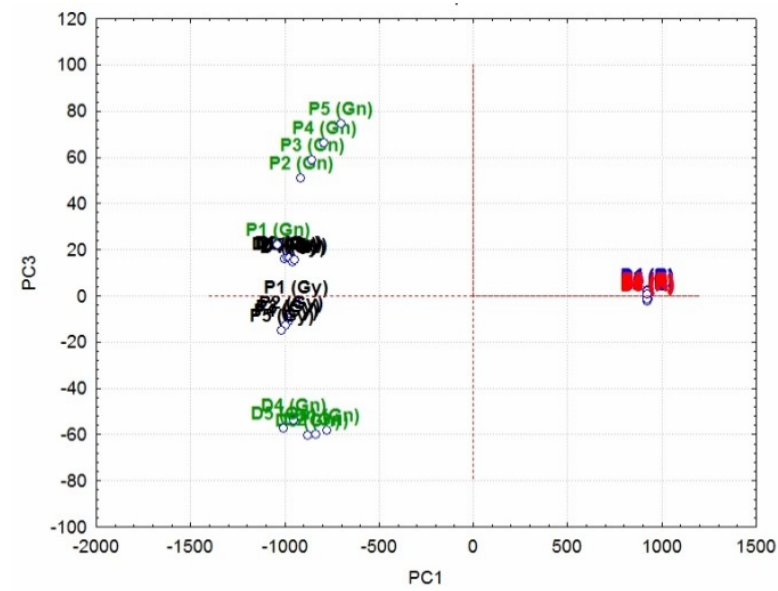

Figure 4. PCA classification of the investigated extracts (according to PC1 vs. PC3 representation) based on chromatographic fingerprints provided by $U V_{254}$ image acquired using Nikon digital camera and green $(G n)$, gray (Gy), blue (B) and red (R) scale selection for image processing.

According to the PC1 vs. PC2 representation (Fig. 5), it could be observed a differentiation of information provided by red and blue scales and also an improved quantity of information provided by green and gray scales respectively. As previously observed, on the green scale the samples are classified according to their provenience (commercially manufacturers).

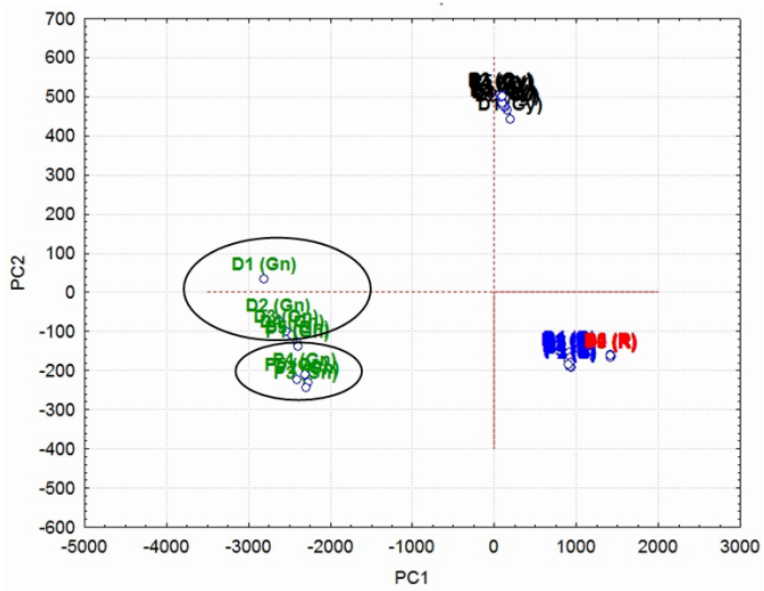

Figure. 5. PCA classification of the investigated extracts (according to PC1 vs. PC2 representation) based on chromatographic fingerprints provided by $\mathrm{UV}_{254}$ image acquired using TLC scanner device and green, gray, blue and red scale selection for image processing. 
In case of the plate documented under UV at $\lambda=366 \mathrm{~nm}$ both images acquired using digital camera and UV-Vis TLC scanner device respectively provided significant information related to the investigated samples.

The PCA investigations on the data matrices provided by digital camera images revealed that the first eight PCs explain more than $99.48 \%$ of the data variability. The PC1 variable (accounting for $82.26 \%$ of data variability) was associated with the area of the plate corresponding to $\mathrm{R}_{\mathrm{f}}=0.37$ while the PC2 and PC3 variables (accounting for $13.35 \%$ of data variability and $1.75 \%$ respectively) were associated with the areas that correspond to a $R_{f}$ values of 0.39 and 0.93 respectively. In this case, the PC1 vs. PC2 representation shows a good separation between information provided by each scale (Fig. 6).

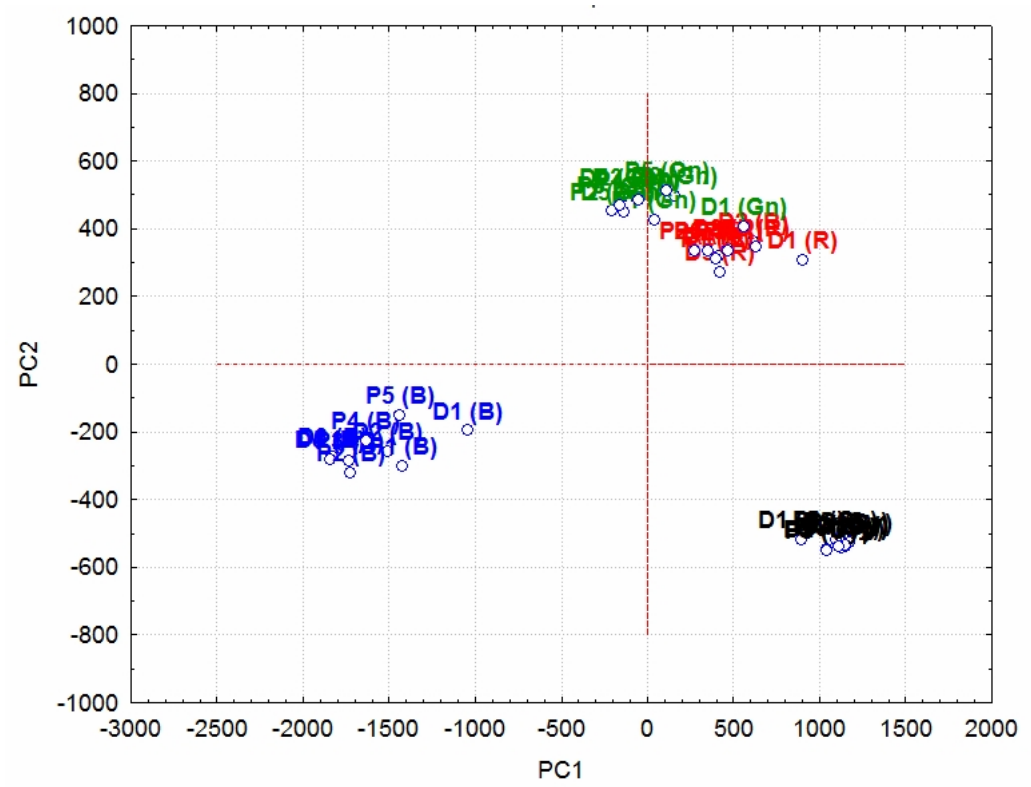

Figure 6. PCA classification of the investigated extracts (according to PC1 vs. PC2 representation) based on chromatographic fingerprints provided by $U_{366}$ image acquired using Nikon digital camera and green $(G n)$, gray (Gy), blue (B) and red $(\mathrm{R})$ scale selection for image processing.

Moreover, according to the PC4 contribution (accounting for $0.66 \%$ of data variability) a good differentiation of the investigated samples based on the extraction system composition was achieved when grey and green scale were used (Fig. 7) 


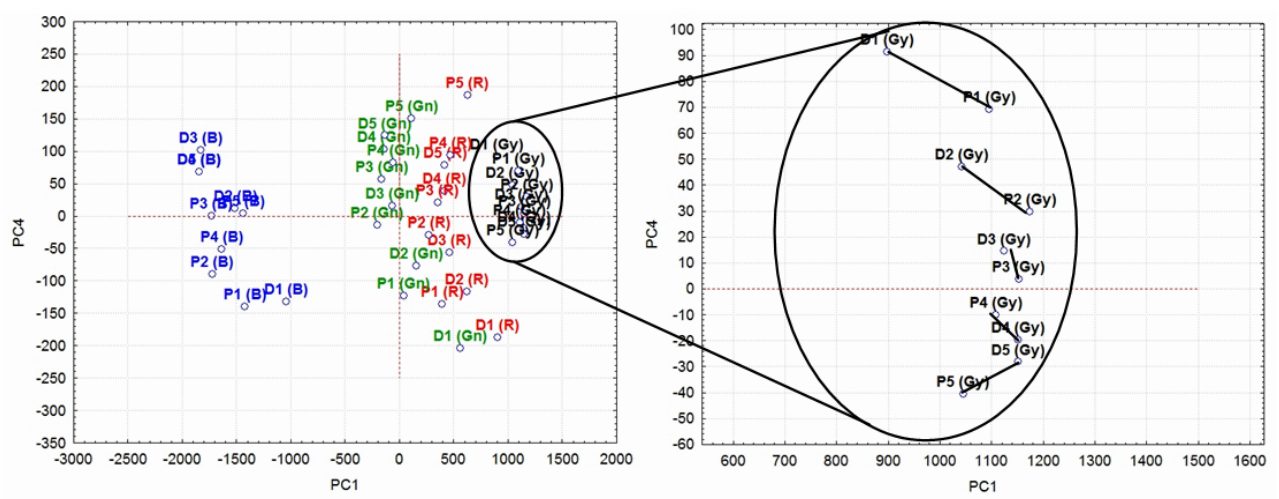

Figure 7. PCA classification of the investigated extracts (according to PC1 vs. PC4 representation) based on chromatographic fingerprints provided by $\mathrm{UV}_{366}$ image acquired using Nikon digital camera and green $(\mathrm{Gn})$, gray $(\mathrm{Gy})$, blue $(\mathrm{B})$ and red (R) scale selection for image processing.

Similar results were obtained based on PCA investigations on data matrices provided by images acquired using UV-Vis TLC scanner device and UV light at $\lambda=366 \mathrm{~nm}$ for chromatographic plate documentation (Fig. 8). In this case the PC1 variable (accounting for $60.55 \%$ of data variability) was associated with the area of the plate corresponding to $R_{f}$ values 0.36 while the PC2 variables (accounting for $32.45 \%$ ) was associated with the area of the plate corresponding to $R_{f}$ values of 0.94 .

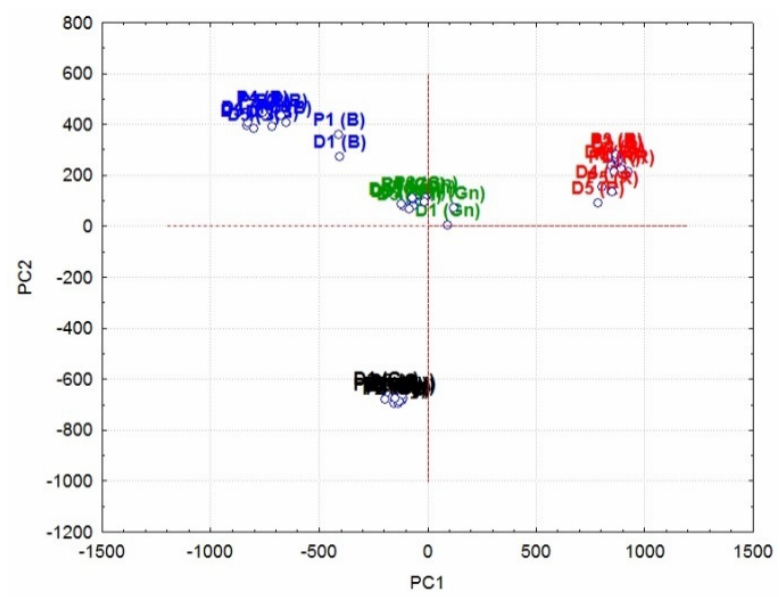

Figure 8. PCA classification of the investigated extracts (according to PC1 vs. PC2 representation) based on chromatographic fingerprints provided by $U_{366}$ image acquired using TLC scanner device and green (Gn), gray (Gy), blue (B) and red (R) scale selection for image processing. 
Considering PC3 which represents $2.48 \%$ of data variability, the graphical representation of PC1 vs. PC3 revealed the tendency of samples differentiating based on the composition of the extraction system (Fig. 9).
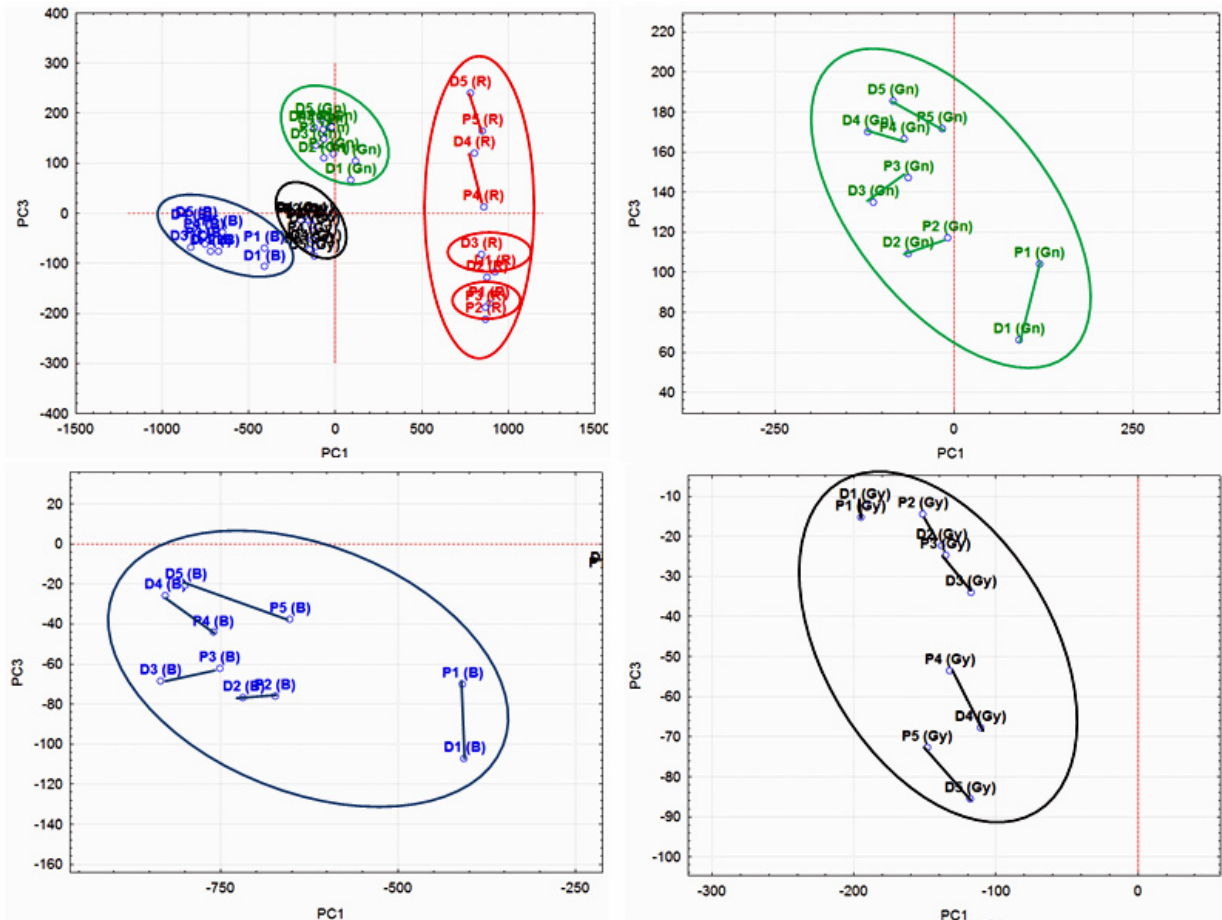

Figure 9. PCA classification of the investigated extracts (according to PC1 vs. PC3 representation) based on chromatographic fingerprints provided by $\mathrm{UV}_{366}$ image acquired using TLC scanner device and green (Gn), gray (Gy), blue (B) and red (R) scale selection for image processing.

Apart of gray, green and blue scale, the red one provides a mixed classification of the extracts: by producer for extract 1-3 (D1-D2-D3 and P1P2-P3) and by extraction solvent composition for extracts 4 and 5 (D4-P4; D5-P5).

Based on the above observations it can be concluded that the information provided by images acquired under UV light at $\lambda=254 \mathrm{~nm}$ processed on green and gray scale are suitable for samples differentiation by their origin while the information provided by images acquired under UV light at $\lambda=366 \mathrm{~nm}$ and processed on green, gray and blue scale are appropriate for samples classification according to the composition of the system used for extraction procedure. 


\section{CONCLUSIONS}

Using various hydroalcoholic extracts from dray vegetal material of Gallium verum plant as test samples for investigations, important aspects related to the HPTLC-IA technique combined with PCA were highlighted.

Based on the obtained results it could be concluded that for image acquisition procedure each of the investigated systems (advanced digital camera and UV-Vis TLC scanner device) were able to provide sufficient information for fingerprint investigation.

The images acquired under UV light at both $\lambda=254 \mathrm{~nm}$ and respectively at $\lambda=366 \mathrm{~nm}$ provides complementary information which are necessary for a complete characterization of complex matrices such as those of extracts from natural plants.

Good differentiation of vegetal samples having different origin (different manufacturers) was obtained by PCA analysis of the chromatographic data provided by fingerprints on UV254 images using green and gray scale selection for chromatograms processing. Classification of extracts according to the composition of extraction system can be achieved using $U V_{366}$ image processing on green, gray and blue scale selection.

HPTLC-IA methodology combined with the PCA technique can be considered a powerful tool in the characterization, authentication, and quality evaluation of extracts from natural plants.

\section{EXPERIMENTAL SECTION}

\section{Material and methods}

\section{Reagents and chemicals}

Analytical grade organic solvents ethanol, ethyl acetate, formic acid and toluene were purchased from Merck (Darmstadt, Germany). Chromatographic separations were carried out on HPTLC Silca gel G $60 F_{254}(20 \times 10)$ plates purchased from Merck (Darmstadt, Germany). NTS reagent (2-aminoethyl diphenylborinate, 98\%) was from Alfa Aesar (Karlsruhe, Germany).

\section{Sample preparation and HPTLC developments}

Gallium verum dry plant for infusion, from Dacia and respectively Plafar manufacturers (Romania), was milled and sieved. The fine powder fractions, with particle size $d_{p}<250 \mu \mathrm{m}$, were used for extraction procedure. The extracts were obtained by 15 days maceration at room temperature using $0.5 \mathrm{~g}$ of powder material and $25 \mathrm{~mL}$ hydroalcoholic mixtures of ethanol - water in different ratio 
$(100: 0 ; 90: 10 ; 80: 20 ; 70: 30 ; 60: 40, \mathrm{v} / \mathrm{v})$. All the obtained extracts were separated from the vegetal material by filtration and stored protected from light at $4{ }^{\circ} \mathrm{C}$ temperature. Volumes of $20 \mu \mathrm{L}$ from each extract were applied on HPTLC Silica gel $60 \mathrm{~F}_{254}$ plate $(20 \times 10 \mathrm{~cm})$ as $10 \mathrm{~mm}$ band using the Linomat 5 TLC applicator (Camag, Muttenz, Switzerland) with an application rate of $60 \mathrm{~nL} / \mathrm{s}$. The chromatographic separation was performed in the normal chamber $20 \times$ $10 \mathrm{~cm}$ (CAMAG, Muttenz, Switzerland) with a mixture of ethyl acetate - toluene formic acid - wather (30:1.5:4:3, v/v/v/v) up to a migration distance of $8 \mathrm{~cm}$ (from the lower plate edge). After the plate was dried their documentation was first performed under UV light at $254 \mathrm{~nm}$ (fluorescence quenching) and then sprayed with NTS solution and documented under UV light at $366 \mathrm{~nm}$.

\section{Image acquisition and processing}

In both cases of visualising, the plate image was captured in two ways; using an advanced digital camera (Nikon D3100, Nikon Corp., Japan, image size of $1922 \times 952$ pixel) and a specialized UV-Vis TLC scanner device (the second-generation instrument for quantitative measurements in TLC, equipped with high qualified Micortek 3-linear color CCD camera with a resolution of 300DPI, BioDit Technology, Co.) respectively. For the multivariate analysis of HPTLC data, the RGB images of chromatograms visualized by fluorescence quenching $\left(\mathrm{UV}_{254}\right.$ image) and under $366 \mathrm{UV}$ light after spraying with NTS $\left(U V_{366}\right.$ image) were converted into gray $(G y)$, green $(G n)$, blue $(B)$ and red $(R)$ pure color scales. The obtained images were further processed with the TLC Analyser software (http://www.sciencebuddies.org/science-researchpapers/tlc_analyzer.shtml) and transformed into numerical data.

\section{Multivariate data analysis}

Among various computational chemometric methods, especially those classified as multivariate exploratory techniques are used to extract systematic information often dispersed over large sets of data. The principal component analysis (PCA), a linear dimensionality reduction technique is the most employed in many chromatographic investigations [27-29]. This is because of its capacity to reduce the dimensionality of the original dataset by retaining the maximum variability of a large number of variables by few underlying factors (principal components - PCs) which explain most of the data variability without losing the important information. In this case the data matrix was composed of numerical values (as independent variables) related to intensities on separated chromatographic bands corresponding to well defined $R_{F}$ values calculated according to the total units considered from the start to front of the plate. For PCA investigation developed on the obtained HPTLC data the Statistica 8.0 (StatSoft, Inc. 1984-2007, Tulsa, USA) software package was used. 
IMAGE ANALYSIS APPROACHES TO IMPROVE THE THIN LAYER CHROMATOGRAPHY ...

\section{ACKNOWLEDGMENTS}

This work was possible with the financial support offered by Romanian Ministry of Education, Research, Youth and Sports through research project PNII-ID-PCE-2011-3-0366.

\section{REFERENCES}

1. B. Hemmateenejad, N. Mobaraki, F. Shakerizadeh-Shirazi, R. Miri, Analyst, 2010, 135, 1747.

2. L.A. Fazakas, R.D. Nascu-Briciu, C. Sârbu, Journal of Liquid Chromatography \& Related Technologies, 2011, 34, 2315.

3. M. Olech, Ł. Komsta, R. Nowak, Ł. Cieśla, M. Waksmundzka-Hajnos, Food Chemistry, 2012, 132, 549.

4. C. Tistaert, B. Dejaegher, Y.V. Heyden, Analytica Chimica Acta, 2011, 690, 148.

5. K. Misra, R. Tulsawani, R. Shyam, D.K. Meena, G. Morlock, Journal of Liquid Chromatography \& Related Technologies, 2012, 35, 1364.

6. M. Gupta, M. Singh, H.M. Mukhatr, S. Ahmad, Pharmacognosy Journal, 2010, 2, 381.

7. H. Wagner, R. Bauer, D. Melchart, P.-Gen Xiao, A. Staudinger, Chromatographic fingerprint analysis of herbal medicines. Thin-layer and High Performance Liquid Chromatography of chinese drugs, Vol. 1, Second Revised and Enlarged Edition, Springer, Wien, New York, 2011.

8. R. Tian, P. Xie, H. Liu, Journal of Chromatography A, 2009, 1216, 2150.

9. D. Milojkovíc-Opsenica, P. Ristivojevíc, F. Andríc, J. Trifkovíc, Chromatographia, 2013, 76, 1239.

10. I.A. Sima (Tuhuţiu), D. Casoni, C. Sârbu, Talanta, 2013, 114, 117.

11. P. Ristivojević, J. Trifković, I. Vovk, D. Milojković-Opsenica, Talanta, 2017, 162, 72.

12. A. Bansal, V. Chhabra, R.K. Rawal, S. Sharma, Journal of Pharmaceutical Analysis, 2014, 4, 223.

13. N. Popović, J. Sherma, Trends in Chromatography, 2014, 9, 21.

14. R.T. Tian, P.S. Xie, H.P. Liu, Journal of Chromatography A, 2009, 1216, 2150.

15. G.E. Morlock, P. Ristivojevic, E.S. Chernetsova, Journal of Chromatography A, 2014, 1328, 104.

16. K.H. Wong, V. Razmovski-Naumovski, K.M. Li, G.Q. Li, K. Chan, Journal of Pharmaceutical and Biomedical Analysis, 2014, 95, 11.

17. P.S. Xie, S. Sun, S. Xu, L. Guo, Journal of Chromatography \& Separation Techniques, 2014, 5, 249.

18. T. Tang, W. Guo, Y. Xu, S. Zhang, X. Xu, D. Wang, Z. Zhao, L. Zhua, D. Yang, Phytochemical Analysis, 2014, 25, 266. 
19. P. Ristivojević, F.Lj Andrić, J.Đ. Trifković, I. Vovk, L.Ž. Stanisavljević, Ž.Lj Tešić, D.M. Milojković-Opsenica, Journal of Chemometrics, 2014, 28, 301.

20. G.E. Morlock, P. Ristivojevic, E.S. Chernetsova, Journal of Chromatography A, 2014, 1328, 104.

21. J.M. Bosque-Sendra, L. Cuadros-Rodriguez, C. Ruiz-Samblas, A. Paulina de la Mata, Analytica Chimica Acta, 2012, 724, 1.

22. H. Atmaca, E. Bozkurt, M. Cittan, H.D. Tepe, Journal of Ethnopharmacology, 2016, $186,305$.

23. N. Chaher, S. Krisa, J.C. Delaunay, S. Bernillon, E. Pedrot, J.M. Mérillon, D. Atmani, T. Richard, Journal of Pharmaceutical and Biomedical Analysis, 2016, $117,79$.

24. M. Tamas, D. Stana, S. Timis, Notulae Botanicae Horti Agrobotanici ClujNapoca, 2006, 34, 1842.

25. L.O. Demirezer, F. Urbuz, Z. Guvenalp, K. Stroch, A. Zeeck, Turkish Journal of Chemistry, 2006, 30, 525.

26. A. Chevallier, Enciclopedia of Herbal Medicine, Ed. DK Penguin Random House, 2016, 214.

27. D. Casoni, C. Sârbu, Spectrochimica Acta Part A: Molecular and Biomolecular Spectroscopy, 2014, 118, 343.

28. A. Kume, S. Kawai, R. Kato, S. Iwata, K. Shimizu, H. Honda, Journal of Bioscience and Bioengineering, 2016, 20, 1.

29. K.K. Vasan, B. Surendiran, Perspectives in Science, 2016, 8, 510. 